\title{
How Capsule Endoscopy Has Illumened the Prevalence of NSAID-Related Distal Small Intestinal Ulceration
}

\author{
Foong Way David Tai ${ }^{1}$
}

Accepted: 6 September 2020 / Published online: 19 September 2020

(c) Springer Science+Business Media, LLC, part of Springer Nature 2020

The commonly prescribed analgesic and anti-inflammatory medications termed nonsteroidal anti-inflammatory drugs (NSAIDs) induce gastroduodenal ulcerations that in turn are well recognized as the basis for upper gastrointestinal (GI) bleeding and perforation. Though these complications are effectively treated and prevented by the use of protonpump inhibitors (PPIs), less well appreciated are the ulcerating effects of NSAIDs on the small intestine distal to the second part of the duodenum. Due to the inhibition of the inducible enzyme cyclooxygenase (COX)-2, NSAIDs reduce the production of mucosal prostaglandin E2, a substance responsible for the regulation of the small intestinal microcirculation and the integrity of the mucus barrier [1], while alterations of small intestinal microbiota homeostasis favoring Gram-negative bacteria increase the susceptibility to ulceration in experimental models [2]. The spectrum of endoscopic features of NSAIDs range from mucosal breaks such as erosions and ulcerations to erythema and denuded areas of mucosa [3].

In this issue of Digestive Diseases and Sciences, Lee and collaborators report on the effects of NSAIDs on small intestinal injuries as visualized with wireless capsule endoscopy [4]. In this retrospective single-center study, they analyzed the risk factors associated with the presence of erosive lesions in the small intestines by examining 309 capsule endoscopy examinations performed over a 5 -year period. In a blinded fashion, Lee and colleagues re-reviewed videos adjudicated by a committee of three experienced experts. Lee et al. reported that $45 \%$ of patients investigated mostly for suspected gastrointestinal bleeding had erosions or ulcers, while in comparison in only 36 patients or $11.6 \%$ of complete capsule examinations, a pathological diagnosis associated with intestinal ulceration such as Crohn's disease,

Foong Way David Tai

david.tai@nhs.net

1 Academic Department of Gastroenterology, Royal Hallamshire Hospital, Glossop Road, Sheffield S10 2JF, UK tuberculosis, or Behçet's syndrome had been given. Patients with definite or suspected erosive lesions with no established cause were then compared with other subjects who had no evidence of erosive lesions. Frequent NSAID ingestion within 2 weeks of capsule endoscopy was present in $14 \%$ of this subset. The authors concluded that idiopathic erosions or ulcers as visualized with capsule endoscopy was statistically associated with the use of NSAIDs after adjusting for age and gender (odds ratio 4.2, 95\% confidence interval 1.9-9.5). Among patients with definite erosive lesions, as determined by agreement of at least two of three blinded reviewers, over half had a history of NSAID ingestion.

Idiopathic small intestinal mucosal breaks are common; prospective trials examining the effects of NSAIDs on small intestinal mucosal injury have excluded as many as $12-14 \%$ of participants undergoing baseline capsule endoscopy with at least one mucosal break prior to randomization [5, 6]. This suggests that occasional mucosal injuries may be a normal finding, particularly in the terminal ileum. On the other hand, the use of NSAIDs is also commonly undeclared. In one study, $13 \%$ of patients undergoing capsule endoscopy were positive for NSAIDs and salicylates as assessed by urinalysis, although only 1/76 participants reported NSAID consumption [7]. With this in mind, the study by Lee and collaborators adds to a body of literature that suggests that up to $78 \%$ of patients taking NSAIDs for $\leq 2$ weeks have endoscopic evidence of small intestinal mucosal injury [3]. Although the study by Lee does not address the chronicity of NSAID consumption beyond 2 weeks, the endoscopic prevalence of mucosal injury is 50-68\% with $>3$ months of NSAIDs [8]. Erosions and ulceration are more common with short term use [8]. Therefore, since the prevalence of NSAID enteropathy in the present study did not examine the more chronic features of NSAID consumption such as erythematous or denuded mucosa, it may underestimate the extent of enteropathy by only reporting ulcerative lesions. In instances where longer-term NSAID use is necessary, the use of selective COX-2 inhibitors may offer some advantage, 
with studies suggesting fewer instances of small intestinal mucosal injury on endoscopy [5, 6, 9]. Protection from mucosal injury persists even when selective COX-2 inhibitors are compared with non-selective agents and PPIs in combination [5, 6]. Nonetheless, selective COX-2 inhibitors have lost favor as a mucosal sparing agent among physicians, after rofecoxib and valdecoxib were withdrawn from the market due to adverse cardiovascular events, instead favoring a non-selective NSAID and concomitant PPI strategy [10]. In fact, the occurrence of small intestinal mucosal injury with NSAIDs may also be exacerbated by the concomitant use of PPIs for gastroduodenal protection [11]. This association was not found in Lee's study as only $1.8 \%$ of study participants were taking PPIs, but is an important consideration.

Perhaps more relevant than the presence of ulceration in suspected NSAID enteropathy are the clinical implications of such endoscopic disease which are difficult to assess outside of a prospective study. Do the small intestinal erosions and ulcerations lead to clinically appreciable bleeding? Suspected GI bleeding was the indication for capsule endoscopy in $71 \%$ of the cohort in Lee's study although evidence of overt or recent bleeding were seen in only $19 \%$. Nevertheless, NSAID consumption may be a more common cause of occult bleeding in some as demonstrated by two large clinical trials of patients randomized to celecoxib, or a non-selective NSAID with [12] or without [13] concomitant PPIs. They suggest that an occult fall in hemoglobin $>2 \mathrm{~g} / \mathrm{L}$, potentially of small intestinal source, were the most common clinically significant events in $>60 \%$ of participants reaching the primary endpoint. Overall, occult GI bleeding from NSAIDs was uncommon (0.4-2.3\%), significantly favoring celecoxib. The significance of the majority of erosive and ulcerative small intestinal lesions commonly observed with capsule endoscopy remains to be determined, and also whether aggressive repletion of iron stores may suffice in a subset of patients.

Lee and colleagues demonstrate that seemingly idiopathic ulcerative lesions in the small intestines are common and are frequent in patients taking NSAIDs. The further appreciation of the clinical significance of such ulcerative lesions, the relative effects of COX-2 selectivity, and the risk and benefits of PPIs have important implications on future management strategies.

Financial Support None.

\section{Compliance with Ethical Standards}

Conflict of interest Authors declare that they have no conflict of interest.

\section{References}

1. Tanaka A, Matsumoto M, Hayashi Y, Takeuchi K. Functional mechanism underlying cyclooxygenase-2 expression in rat small intestine following administration of indomethacin: relation to intestinal hypermotility. J Gastroenterol Hepatol. 2005;20:38-45.

2. Uejima M, Kinouchi T, Kataoka K, Hiraoka I, Ohnishi Y. Role of intestinal bacteria in ileal ulcer formation in rats treated with a nonsteroidal antiinflammatory drug. Microbiol Immunol. 1996;40:553-560.

3. Tai FWD, McAlindon ME. NSAIDs and the small bowel. Curr Opin Gastroenterol. 2018;34:175-182.

4. Lee SP et al. Effect of nonsteroidal anti-inflammatory agents on small intestinal injuries as evaluated by capsule endoscopy. Dig Dis Sci. (Epub ahead of print). https://doi.org/10.1007/s1062 0-020-06511-z.

5. Goldstein JL, Eisen GM, Lewis B, et al. Video capsule endoscopy to prospectively assess small bowel injury with celecoxib, naproxen plus omeprazole, and placebo. Clin Gastroenterol Hepatol. 2005;3:133-141.

6. Goldstein JL, Eisen GM, Lewis B, et al. Small bowel mucosal injury is reduced in healthy subjects treated with celecoxib compared with ibuprofen plus omeprazole, as assessed by video capsule endoscopy. Aliment Pharmacol Ther. 2007;25:1211-1222.

7. Sidhu R, Brunt LK, Morley SR, Sanders DS, McAlindon ME. Undisclosed use of nonsteroidal anti-inflammatory drugs may underlie small-bowel injury observed by capsule endoscopy. Clin Gastroenterol Hepatol. 2010;8:992-995.

8. Maiden L. Capsule endoscopic diagnosis of nonsteroidal antiinflammatory drug-induced enteropathy. J Gastroenterol. 2009;44:64-71.

9. Laine L, Smith R, Min K, Chen C, Dubois RW. Systematic review: the lower gastrointestinal adverse effects of non-steroidal antiinflammatory drugs. Aliment Pharmacol Ther. 2006;24:751-767.

10. Sun SX, Lee KY, Bertram CT, Goldstein JL. Withdrawal of COX-2 selective inhibitors rofecoxib and valdecoxib: impact on NSAID and gastroprotective drug prescribing and utilization. Curr Med Res Opin. 2007;23:1859-1866.

11. Washio E, Esaki M, Maehata Y, et al. Proton pump inhibitors increase incidence of nonsteroidal anti-inflammatory druginduced small bowel injury: a randomized, placebo-controlled trial. Clin Gastroenterol Hepatol. 2016;14:809-15e1.

12. Chan FK, Lanas A, Scheiman J, Berger MF, Nguyen H, Goldstein JL. Celecoxib versus omeprazole and diclofenac in patients with osteoarthritis and rheumatoid arthritis (CONDOR): a randomised trial. Lancet. 2010;376:173-179.

13. Cryer B, Li C, Simon LS, Singh G, Stillman MJ, Berger MF. GI-REASONS: a novel 6-month, prospective, randomized, open-label, blinded endpoint (PROBE) trial. Am J Gastroenterol. 2013;108:392-400.

Publisher's Note Springer Nature remains neutral with regard to jurisdictional claims in published maps and institutional affiliations. 